\title{
Análisis de Valores y Actitudes en Temas Morales en Estudiantes de Educación Secundaria
}

\section{Analysis of Values and Attitudes to Moral Issues in Middle School Students}

\author{
Francisco Manuel Morales Rodríguez y María Victoria Trianes Torres \\ Universidad de Málaga, España
}

\begin{abstract}
Resumen. El objetivo del presente estudio es aportar una evaluación sobre valores y actitudes en temas morales en una muestra de estudiantes de educación secundaria analizando diferencias en función del sexo y de la edad. Los participantes han sido 450 estudiantes de Educación Secundaria Obligatoria, con edades comprendidas entre 12 y 15 años. La mayoría de los encuestados están muy de acuerdo con los aspectos referidos a si la situaciones planteadas pueden ser consideradas robar o una acción violenta. Pocos participantes realizan actos solidarios. Se aprecia mayor tendencia a la despenalización de la violencia si es en defensa de lo propio y menor predisposición a conductas prosociales en chicos y adolescentes de menor edad. Para concluir, se destaca la importancia de una detección y evaluación temprana de actitudes e intereses hacia estos temas morales con vistas a una educación integral dirigida a la mejora de la convivencia social.

Palabras clave: estudiantes de educación secundaria, evaluación de valores y actitudes.
\end{abstract}

\begin{abstract}
The objective of this study is to provide an assessment of values and attitudes towards moral issues in a sample of middle school students, by analyzing differences according to gender and age. The participants were 450 middle school students between 12 and 15 years old. The majority of the interviewees strongly agree that the situations presented to them may be considered stealing, or violent acts. Few students were found to carry out actions of solidarity. A greater tendency to decriminalize violence can be seen if it is in self-defense. Similarly, there is less of a tendency towards prosocial behavior in the boys and the younger children. In conclusion, the study highlights the importance of detection and early evaluation of attitudes and interests in the case of these moral issues, with a view to a comprehensive education aimed at the improvement of social coexistence.

Keywords: middle school students, assessment of values and attitudes.
\end{abstract}

\section{Introducción}

La educación moral y concretamente en lo que a valores y actitudes se refiere constituye uno de los temas recientes que figura en los textos legales y en las sucesivas reformas del sistema educativo en todas sus etapas de un modo u otro. En relación con estas cuestiones se han introducido importantes novedades pero con el denominador común y objetivo general de conseguir que la formación moral tenga un papel cada vez más necesario, relevante e imprescindible en el conjunto de actividades educativas en nuestros colegios, IES, universidades e instituciones en los tiempos que nos toca vivir.

La violencia escolar se manifiesta cada día en nuestros centros educativos con consecuencias muy negativas para el alumnado, profesorado y en nuestras sociedades en general. Precisamente esta violencia afecta a la convivencia social y humana que conlleva enfatizar aspectos éticos de los que en muchas ocasiones se ado-

La correspondencia sobre este artículo debe enviarse al primer autor ale-mail:framorrod@uma.es lece así como la puesta en práctica de valores como la solidaridad, la libertad, la igualdad, la justicia y la paz. Los centros escolares constituyen el espacio óptimo y dialógico para la construcción en libertad de una sociedad abierta y plural y de unas instituciones que tienen entre sus competencias básicas o transversales la de formar futuros ciudadanos socialmente responsables. Después de la primera década del siglo XXI, la educación en valores como la solidaridad es considerada un factor cada vez más indispensable en las actuales sociedades.

Si bien es cierto que la educación moral no es una preocupación reciente, si lo es la voluntad actual de convertirla en uno de los pilares de la educación. También son, en buena parte, novedosos los instrumentos psicopedagógicos y estrategias didácticas que se proponen para hacer el tratamiento de las cuestiones de valor, además de ser aún pocos los instrumentos de evaluación existentes al respecto. Somos conocedores de que la educación no es aséptica y que el acto de educar implícita o explícitamente contiene valores y de ahí la necesidad de priorizar unos sobre otros. Para González (2002), la “educación para la conviven- 
cia...debe desarrollar el discurso de los llamados valores para la vida y para la convivencia (como elenco de valores derivados de una ética mínima), incidiendo en los grandes y básicos valores de la libertad, de la solidaridad, de la igualdad, de la justicia y de la paz, así como la recuperación y en la defensa de "pequeños valores" que corren el riesgo de pasar a un segundo orden de importancia". Se trata, según este autor, de valores como la honestidad, la responsabilidad, el esfuerzo, el sacrificio, la voluntad, la generosidad, la ternura o la esperanza; valores que Peiró (2009) define como valores propiamente humanos que son los que en realidad pueden hacer posible la realización y la vivencia de los "grandes valores democráticos".

Estas innovaciones subyacentes en este ámbito de los valores parten de un espíritu abierto a las cuestiones valorativas. Los Reales Decretos, Decretos y demás documentos y recursos legislativos que establecen el currículum de la educación primaria y de la educación secundaria obligatoria, concretan la necesidad de asegurar el desarrollo integral del alumnado en estas etapas, y las propias expectativas de la sociedad coinciden en demandar un currículum que no se limite a la adquisición de conceptos y conocimientos académicos vinculados a la enseñanza más tradicional, sino que, además, incluya otros aspectos que ayuden al desarrollo de las personas como tales, ejemplos de estos son las habilidades prácticas, las actividades morales y los valores. La educación social y la educación moral son el fundamento elemental del desarrollo educativo, que debe permitir la asunción de responsabilidades en la sociedad actual y del futuro, una sociedad pluralista, en la que las propias creencias, valoraciones y opciones han de convivir con el respeto y creencias a los valores de los demás.

El cambio en la conceptualización del término transversalidad ha sufrido una evolución rápida, llegando a simbolizar un aspecto que introduce elementos de innovación en el sistema educativo actual en nuestro país. Existe acuerdo entre los autores (Bolívar, 1995; Buxarrais, 1997; Cortés, Hernán y López, 1998; Fouce, 2001; García, 1994; Gervilla, 1997; Lucini, 1996; Touriñán, 2008) en el hecho de que el término transversal hace referencia a la educación moral y cívica, para la salud, para la paz y la convivencia, para la igualdad de oportunidades entre los sexos, del consumidor, ambiental y vial entre otras cuestiones. Lo cierto es que, la concepción del término ha pasado por diferentes momentos con distintos significados, hasta llegar a lo que representa en estos momentos. Diversos sectores del mundo educativo le han ido progresivamente asignando diferentes enunciados intentando, de esta forma, llenar algunas de las lagunas que posee la ya no tan reciente LOGSE, LOU, respecto a este tema no exento de relevancia.

El término transversal no solo puede significar hoy la inclusión de ciertos contenidos que deben considerarse en las diversas disciplinas escolares, para ilustrar una serie de valores y actitudes que deben ser educa- dos sino que conlleva síntomas de innovación y ha sido usado como paradigma incluso de algunas de las citadas Reformas Educativas. Por tanto, en la actualidad los temas transversales son contenidos curriculares que responden, en lo esencial, a las siguientes características: Ilustran una preocupación por los problemas sociales, representando situaciones problemáticas vividas actualmente en nuestras sociedades y que conectan fácilmente con las informaciones, inquietudes y vivencias del propio alumnado. Además conectan el centro educativo con la vida, con la realidad cotidiana. La educación tiene por obligación promover la interacción entre la cultura pública y la cultura experiencial de cada uno de sus alumnos. El centro además ha de hacer posible la síntesis entre entre las capacidades intelectuales del alumnado y sus capacidades afectivas, sociales y éticas. Se trata de una apuesta por la educación en valores, como uno de los ejes fundamentales de la educación integral. La necesidad de la educación moral se ha convertido últimamente en un clamor frente al relativismo moral, a la inhibición de los agentes tradicionales de socialización y a la emergencia de los agentes educativos informales. También permiten adoptar una perspectiva social crítica frente a los currículos tradicionales que, sin duda, obstaculizan las visiones globales e interrelacionadas de los problemas de la comunidad educativa, que tan necesarias son en sociedades y civilizaciones como las actuales.

Puede destacarse que la educación en valores como la solidaridad y la no violencia se afirma en la necesidad de constituirse en el principio rector de toda educación, orientando el desarrollo de los currículum de los diversos niveles educativos en la búsqueda de una formación integral del alumnado, promoviendo actitudes y valores éticos y mostrando, en la programación y vida diaria dicha conformidad con valores éticos y socialmente deseables (Trianes y Fernández, 2001). Por ello, el objetivo general del presente estudio es realizar una evaluación de actitudes e intereses hacia temas morales en una muestra de adolescentes estudiantes de educación secundaria analizando diferencias en función del sexo y de la edad.

\section{Método}

\section{Participantes}

Han participado en este estudio un total de 450 estudiantes de Educación Secundaria Obligatoria, con edades comprendidas entre 12 y 15 años, con una edad media de 13.21 años $(D T=1.19)$ pertenecientes todos ellos a institutos públicos de la provincia de Málaga de ámbito urbano, 243 chicas y 207 chicos. Los institutos participantes en este estudio tienen similares directrices educativas, utilizan los mismos libros, recursos y materiales curriculares. En cuanto a la selección de la muestra además puede señalarse que se ha trabajado con una muestra incidental no probabilística. 
Tabla 1. Valores y actitudes en temas morales (\%)

\section{OPCIONES DE RESPUESTA}

PORCENTAJES

1. Si un adolescente que entra en un Centro comercial, coge una cinta de vídeo, la esconde y se la lleva sin pagar ¿Es eso robar? ¿Por qué?

$1=$ Sí, porque no ha pagado

$93 \%$

$2=$ No, porque no hace ningún mal

$2 \%$

$3=$ No, porque en los grandes almacenes no se dan cuenta

$5 \%$

2. Si un joven que está comprándose su casa, trata de no pagar impuestos ocultando parte de sus ingresos. ¿Es eso robar?

$1=$ No, ya que es su dinero y lo ha ganado el

$17 \%$

2 = Sí, porque hay que pagar los impuestos entre todos para que el gobierno pueda hacer carreteras, escuelas, hospitales, etc.

3. Si una persona descubre a un asaltante que había entrado en su casa a robar y le pega un tiro. ¿Es una acción violenta?

$1=$ Sí

$86 \%$

$2=$ No

$14 \%$

\section{1. ¿Es justa la acción señala ¿Es una acción justa y por qué?}

1 = Sí, porque ha entrado a robar y que lo pague

$2=$ No, porque no se puede matar

$12 \%$

3 = Sí, porque el ladrón podría haberle agredido

$16 \%$

4 = No, porque podría haber buscado otra solución (llamar a la policía o amenazarlo)

$47 \%$

$5=$ No, porque por entrar a robar no merece nadie ser tiroteado

4. Si un estudiante al salir del instituto recibe una paliza por parte de un grupo y, en respuesta, saca una navaja con la que hiere a uno de ellos y lo mata. ¿Es una acción violenta?

$1=$ Sí $^{\prime}$

$87 \%$

$2=$ No

4.1. ¿Es la acción anteriormente ¿Es dicha acción justa y por qué?

$1=$ Sí, porque es en defensa propia

$2=$ No, porque es en defensa propia

$3=$ No, porque matar es una respuesta desproporcionada

\section{Si una persona se acerca a ti pidiéndote dinero cuando vas de compras. ¿Sientes que debes dárselo y por qué?}

$1=$ Sí, porque lo necesita

$2=$ No, porque no es mi responsabilidad

3 = Sí, porque hay que compartir lo que uno tiene

$4=$ No, porque lo que tiene que hacer es buscar trabajo.

$5=$ Sí, porque me dan pena

$6=$ No, porque lo quieren para malgastárselo en alcohol, drogas, etc

6. Cuando ves la propaganda de las ONGs pidiendo ayuda o dinero para proyectos con colectivos desfavorecidos ¿Siente que debe ayudar y por qué?

$1=$ Sí, porque me dan pena

$2=$ No,porque se quedan con el dinero y no llega a los pobres

$18 \%$

$3=$ Sí, porque hay que ser solidarios y compartir

$68 \%$

4 = No, porque se da dinero a las ONG`s y nadie da trabajo a los pobres

7. Cuando ves que en tu entorno (familiar, de amigos, compañeros de clase, etc) se va a producir un conflicto. ¿Intentas evitarlo y por qué?

$1=$ Sí, porque no me gusta que haya conflictos 
Tabla 1. Valores y actitudes en temas morales (\%) (Continuación)

\section{OPCIONES DE RESPUESTA}

8. ¿Es justa la pena de muerte? ¿Por qué?

$1=$ Sí, porque el que la hace la paga

$21 \%$

$2=$ No, porque no se debe matar a nadie

$79 \%$

9. Cuando el ayuntamiento da viviendas gratuitas a personas que han sufrido una tragedia que les ha dejado sin hogar. ¿Estás de acuerdo con esa medida? ¿Por qué?

$1=$ Sí, porque lo han perdido todo en una tragedia

$37 \%$

2 = No, porque hay muchas más gente que también lo necesita

$13 \%$

$3=$ Sí, porque debemos ayudar a los que han sufrido una desgracia

$50 \%$

10. ¿Estás de acuerdo con que Europa cierre sus fronteras a los inmigrantes de países con menos recursos? ¿Por qué?

$1=$ Sí, porque nos quitan oportunidades

$6 \%$

$2=$ No, porque todos tienen derechos (vivir donde quieran, buscar un trabajo, etc)

$75 \%$

$3=$ Sí, porque traen conflictos

$4=$ No, porque hay trabajo para todos

$15 \%$

$5=$ No, porque aumentan las riquezas del país

$3 \%$

$1 \%$

11. ¿Colaboras o participas en alguna organización humanitaria no gubernamental?

$1=$ Sí

$23 \%$

$2=\mathrm{No}$

$77 \%$

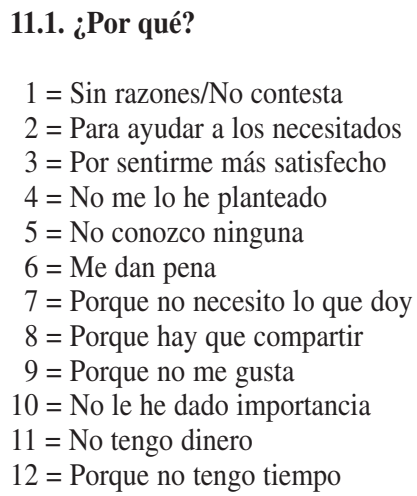

$22.7 \%$

$16.1 \%$

$5.1 \%$

$12.9 \%$

$1.9 \%$

$3.8 \%$

$4.5 \%$

$10.3 \%$

$1.2 \%$

$3.2 \%$

$5.8 \%$

$12.2 \%$

\section{Instrumentos}

"Cuestionario sobre valores y actitudes en temas morales, VATM": Este cuestionario consta de un total de 13 ítems, agrupados en 11 cuestiones en los que se le proporciona al sujeto un enunciado o se les plantea una situación conflictiva para que elija entre distintas alternativas de respuesta (versión inicial más cualitativa) y además se ha utilizado otra versión del mismo cuestionario en formato de respuesta tipo Likert donde los estudiantes evalúan en una escala de 1 a 4 el grado de acuerdo con las cuestiones planteadas (siendo $1=$ Muy en desacuerdo y $5=$ Muy de acuerdo). Presenta adecuadas propiedades psicométricas, siendo el índice de consistencia interna para la escala total obtenido mediante el cálculo del coeficiente Alpha ()$=.68$. Evalúa algunas cuestiones actitudinales personales como el robo, la solidaridad, la cooperación, la ayuda internacional, los derechos humanos, etc. Se trata de un cuestionario de tipo actitudinal para investigar estas cuestiones de máxima candencia.

\section{Procedimiento}

Los datos fueron recogidos por dos orientadores y un investigador de la UMA en los distintos centros de Educación Secundaria participantes en este estudio. El cuestionario fue cumplimentado en las clases ordinarias de los distintos centros de estudios que han participado en este estudio tras gestionar las correspondientes autorizaciones, con similar política educativa en lo que a la dirección de sus centros de estudio se refiere. Se solicitaron por escrito las correspondientes autorizaciones tanto de las familias, del profesorado y de los Jefes de Estudio correspondientes a cada centro, deli- 
Tabla 2. Diferencia en actitudes, valores e intereses en temas morales según sexo

\begin{tabular}{|c|c|c|c|c|}
\hline & Sexo & Media & Desviación típica & $t$ \\
\hline \multirow{2}{*}{$\begin{array}{l}\text { 1. Si un adolescente entra en un centro comercial, cogen una cinta } \\
\text { de vídeo, la esconde y se la lleva sin pagar. ¿Es eso robar? }\end{array}$} & Hombre & 3.67 & .75 & \\
\hline & Mujer & 3.87 & .52 & $-3.59^{*}$ \\
\hline \multirow{2}{*}{$\begin{array}{l}\text { 2. Si un joven que está comprándose su casa trata de no pagar } \\
\text { impuestos ocultando parte de sus ingresos. ¿Es eso robar? }\end{array}$} & Hombre & 3.16 & .86 & \\
\hline & Mujer & 3.33 & .77 & $-2.30 * *$ \\
\hline \multirow{2}{*}{$\begin{array}{l}\text { 3. Si una persona descubre a un asaltante que había entrado } \\
\text { a su casa a robar y le pega un tiro. ¿Es una acción violenta? }\end{array}$} & Hombre & 3.13 & 1.01 & \\
\hline & Mujer & 3.37 & .86 & $-2.83^{*}$ \\
\hline \multirow[t]{2}{*}{ 3.1. ¿Es justa dicha acción? } & Hombre & 2.29 & .96 & \\
\hline & Mujer & 2.14 & .97 & 1.71 \\
\hline \multirow{2}{*}{$\begin{array}{l}\text { 4. Si un estudiante al salir del instituto recibe una paliza } \\
\text { por parte de un grupo y, en respuesta, saca una navaja con la } \\
\text { que hiere a uno de ellos y lo mata. ¿Es una acción violenta? }\end{array}$} & Hombre & 3.31 & .94 & \\
\hline & Mujer & 3.55 & .76 & $-3.01^{*}$ \\
\hline \multirow[t]{2}{*}{ 4.1. ¿Es justa dicha acción? } & Hombre & 2.24 & .94 & \\
\hline & Mujer & 2.07 & .92 & $2.12 * *$ \\
\hline \multirow{2}{*}{$\begin{array}{l}\text { 5. Si una persona se acerca a ti pidiéndote dinero cuando } \\
\text { vas de compras. ¿Sientes que debes dárselo? }\end{array}$} & Hombre & 1.82 & .92 & \\
\hline & Mujer & 2.00 & .89 & $-2.24 * *$ \\
\hline \multirow{2}{*}{$\begin{array}{l}\text { 6. Cuando ves la propaganda de las ONGs pidiendo ayuda } \\
\text { o dinero para proyectos en colectivos desfavorecidos. } \\
\text { ¿Sientes que debes ayudar? }\end{array}$} & Hombre & 2.66 & .82 & \\
\hline & Mujer & 2.99 & .88 & $-4.34 *$ \\
\hline \multirow{2}{*}{$\begin{array}{l}\text { 7. Cuando ves que en tu entorno (familiar, de amigos, compañe- } \\
\text { ros de clase, etc) se va a producir un conflicto. ¿Intentas evitarlo? }\end{array}$} & Hombre & 3.42 & .74 & \\
\hline & Mujer & 3.52 & .78 & -1.35 \\
\hline \multirow[t]{2}{*}{ 8. ¿Es justa la pena de muerte? } & Hombre & 2.01 & 1.02 & \\
\hline & Mujer & 1.88 & .98 & 1.51 \\
\hline \multirow{2}{*}{$\begin{array}{l}\text { 9. Cuando el ayuntamiento da viviendas gratuitas a personas } \\
\text { que han sufrido una tragedia que les ha dejado sin hogar. } \\
\text { ¿Estás de acuerdo con esa medida? }\end{array}$} & Hombre & 3.44 & .80 & \\
\hline & Mujer & 3.64 & .72 & $-3.00 *$ \\
\hline \multirow{2}{*}{$\begin{array}{l}\text { 10. ¿Estás de acuerdo con que Europa cierre sus fronteras } \\
\text { a los inmigrantes de países con menos recursos? }\end{array}$} & Hombre & 2.22 & .91 & \\
\hline & Mujer & 1.95 & .89 & $3.24 *$ \\
\hline \multirow{2}{*}{$\begin{array}{l}\text { 11. ¿Estás dispuesto/a a intervenir si ves alguna acción injusta } \\
\text { para alguien? }\end{array}$} & Hombre & 3.15 & .74 & \\
\hline & Mujer & 3.28 & .78 & $-2.07 * *$ \\
\hline
\end{tabular}

mitando además el horario fijado para el desarrollo de esta actividad.

Además se proporcionó al alumnado las correspondientes instrucciones para la cumplimentación del cuestionario, asegurando además en todo momento la confidencialidad de los datos obtenidos en este estudio y explicando el uso ulterior de las pruebas con fines de investigación.

No se limitó ni espacial ni temporalmente el tiempo destinado a la cumplimentación del cuestionario por parte del alumnado al que se le dio la posibilidad de responder ante posibles dudas y problemas de comprensión al respecto o forma de rellenar el cuestionario propuesto.

Las respuestas en el cuestionario fueron chequeadas y contabilizadas rigurosamente antes de proceder a la introducción de los datos en el SPSS para posterior análisis.

\section{Análisis de datos}

Para el tratamiento estadístico de los datos se usó el paquete estadístico informatizado SPSS 15.0. Se realizaron análisis descriptivos reflejándose porcentajes, medias y desviaciones típicas además de aplicar la prueba $\mathrm{T}$ de Student para muestras independientes para ver si existen diferencias estadísticamente significativas en la evaluación de actitudes e intereses en temas morales en función del sexo y de la edad.

\section{Resultados}

A la vista de los resultados obtenidos se puede, a prio- 
Tabla 3. Diferencia en actitudes, valores e intereses en temas morales según edad

\begin{tabular}{|c|c|c|c|c|}
\hline & Edad & Media & Desviación típica & $t$ \\
\hline $\begin{array}{l}\text { 1. Si un adolescente entra en un centro comercial, cogen una cinta } \\
\text { de vídeo, la esconde y se la lleva sin pagar. ¿Es eso robar? }\end{array}$ & $\begin{array}{l}>13 \\
<=13\end{array}$ & $\begin{array}{l}3.79 \\
3.81\end{array}$ & $\begin{array}{l}.66 \\
.52\end{array}$ & -.39 \\
\hline $\begin{array}{l}\text { 2. Si un joven que está comprándose su casa trata de no pagar } \\
\text { impuestos ocultando parte de sus ingresos. ¿Es eso robar? }\end{array}$ & $\begin{array}{l}>13 \\
<=13\end{array}$ & $\begin{array}{l}3.26 \\
3.27\end{array}$ & $\begin{array}{l}.84 \\
.70\end{array}$ & -.19 \\
\hline $\begin{array}{l}\text { 3. Si una persona descubre a un asaltante que había entrado } \\
\text { a su casa a robar y le pega un tiro. ¿Es una acción violenta? }\end{array}$ & $\begin{array}{l}>13 \\
<=13\end{array}$ & $\begin{array}{l}3.37 \\
3.02\end{array}$ & $\begin{array}{l}.89 \\
.96\end{array}$ & $3.98 *$ \\
\hline 3.1. ¿Es justa dicha acción? & $\begin{array}{l}>13 \\
<=13\end{array}$ & $\begin{array}{l}2.19 \\
2.27\end{array}$ & $\begin{array}{l}.97 \\
.98\end{array}$ & -.89 \\
\hline $\begin{array}{l}\text { 4. Si un estudiante al salir del instituto recibe una paliza } \\
\text { por parte de un grupo y, en respuesta, saca una navaja con la } \\
\text { que hiere a uno de ellos y lo mata. ¿Es una acción violenta? }\end{array}$ & $\begin{array}{l}>13 \\
<=13\end{array}$ & $\begin{array}{l}3.50 \\
3.33\end{array}$ & $\begin{array}{l}.81 \\
.87\end{array}$ & $2.18^{* *}$ \\
\hline 4.1. ¿Es justa dicha acción? & $\begin{array}{l}>13 \\
<=13\end{array}$ & $\begin{array}{l}2.08 \\
2.29\end{array}$ & $\begin{array}{r}.91 \\
.94\end{array}$ & $-2.34 * *$ \\
\hline $\begin{array}{l}\text { 5. Si una persona se acerca a ti pidiéndote dinero cuando } \\
\text { vas de compras. ¿Sientes que debes dárselo? }\end{array}$ & $\begin{array}{l}>13 \\
<=13\end{array}$ & $\begin{array}{l}1.93 \\
1.94\end{array}$ & $\begin{array}{l}.92 \\
.84\end{array}$ & -.09 \\
\hline $\begin{array}{l}\text { 6. Cuando ves la propaganda de las ONGs pidiendo ayuda } \\
\text { o dinero para proyectos en colectivos desfavorecidos. } \\
\text { ¿Sientes que debes ayudar? }\end{array}$ & $\begin{array}{l}>13 \\
<=13\end{array}$ & 2.83 & .88 & -1.28 \\
\hline $\begin{array}{l}\text { 7. Cuando ves que en tu entorno (familiar, de amigos, compañe- } \\
\text { ros de clase, etc) se va a producir un conflicto. ¿Intentas evitarlo? }\end{array}$ & $\begin{array}{l}>13 \\
<=13\end{array}$ & $\begin{array}{l}3.46 \\
3.55\end{array}$ & $\begin{array}{l}.77 \\
.71\end{array}$ & -1.25 \\
\hline 8. ¿Es justa la pena de muerte? & $\begin{array}{l}>13 \\
<=13\end{array}$ & $\begin{array}{l}1.85 \\
2.10\end{array}$ & $\begin{array}{c}.99 \\
1.01\end{array}$ & $-1.56^{* *}$ \\
\hline $\begin{array}{l}\text { 9. Cuando el ayuntamiento da viviendas gratuitas a personas } \\
\text { que han sufrido una tragedia que les ha dejado sin hogar. } \\
\text { ¿Estás de acuerdo con esa medida? }\end{array}$ & $\begin{array}{l}>13 \\
<=13\end{array}$ & $\begin{array}{l}3.59 \\
3.49\end{array}$ & $\begin{array}{l}.73 \\
.82\end{array}$ & 1.39 \\
\hline $\begin{array}{l}\text { 10. ¿Estás de acuerdo con que Europa cierre sus fronteras } \\
\text { a los inmigrantes de países con menos recursos? }\end{array}$ & $\begin{array}{l}>13 \\
<=13\end{array}$ & $\begin{array}{l}1.98 \\
2.23\end{array}$ & $\begin{array}{l}.88 \\
.92 \\
\end{array}$ & $-2.96 *$ \\
\hline $\begin{array}{l}\text { 11. ¿Estás dispuesto/a a intervenir si ves alguna acción injusta } \\
\text { para alguien? }\end{array}$ & $\begin{array}{l}>13 \\
<=13\end{array}$ & $\begin{array}{l}3.25 \\
3.18\end{array}$ & $\begin{array}{l}.72 \\
.69\end{array}$ & 1.02 \\
\hline
\end{tabular}

Significación estadística. ${ }^{*} \mathrm{p}<.01 ; * * \mathrm{p}<.05$

ri, vislumbrar algunas tendencias, que se creen útil comentar relacionando ítems parecidos o similares por su contenido. A continuación en la tabla 1 se presentan los datos resumidos respecto a las actitudes e intereses en temas morales de la muestra de estudiantes de educación secundaria con sus correspondientes porcentajes.

Se puede comprobar que un amplio porcentaje de los estudiantes de Educación Secundaria de la muestra están muy de acuerdo con los aspectos reflejados en los ítems 1, 4 y 7 referidos a si la situaciones planteadas pueden ser consideradas robar, una acción violenta o si tratan de evitar el que se produzca un conflicto $(93.00 \% ; 87.00 \%$ y $80.00 \%$ respectivamente). El $77.00 \%$ de los adolescentes de Educación Secundaria no colabora o participa en alguna ONG; considerando como razones principales para ello la falta de tiempo o que aún no se lo han planteado. Asimismo el 71.24\% tampoco realizan ninguna actividad solidaria señalando como motivos: falta de información al respecto, falta de tiempo, desidia, no se ha presentado la oportunidad y no se lo han planteado, no tener dinero para ello. Puede destacarse que muchos de estos adolescentes consideran como motivo el hecho de que no se han planteado seriamente dicha posibilidad, aún no le han dado importancia, no conocen ninguna ONG o no tienen tiempo ni dinero para ello.

\section{Diferencias en actitudes e intereses en temas morales en función del sexo y de la edad}

A continuación para determinar si existen diferen- 
Tabla 4. Participación en ONGs y realización de actividades solidarias según sexo y edad (\%)

\begin{tabular}{|c|c|c|}
\hline & Sexo & Porcentaje \\
\hline ¿Colabora o participa en alguna organización no gubernamental (ONG)? & $\begin{array}{c}\text { Hombre } \\
\text { Mujer }\end{array}$ & $\begin{array}{l}38.77 \\
60.68\end{array}$ \\
\hline ¿Realiza alguna actividad solidaria, es decir, de ayuda a los demás sin retribución económica? & $\begin{array}{l}\text { Hombre } \\
\text { Mujer }\end{array}$ & $\begin{array}{l}38.76 \\
61.23\end{array}$ \\
\hline & Edad & Porcentaje \\
\hline ¿Colabora o participa en alguna organización no gubernamental (ONG)? & $\begin{array}{c}>13 \\
<=13\end{array}$ & $\begin{array}{l}72.22 \\
27.77\end{array}$ \\
\hline ¿Realiza alguna actividad solidaria, es decir, de ayuda a los demás sin retribución económica? & $\begin{array}{c}>13 \\
<=13\end{array}$ & $\begin{array}{l}71.93 \\
28.06\end{array}$ \\
\hline
\end{tabular}

cias estadísticamente significativas en la variable actitudes e intereses en temas morales en función del sexo y de la edad se han realizado análisis de diferencia de medias (T de Student). La metodología para el análisis de las diferencias según sexo y edad es de carácter cuantitativa, utilizando el cuestionario en formato de respuesta tipo Likert de cuatro puntos $(1=$ Muy en desacuerdo y 4 = Muy de acuerdo). Para este análisis de medias la edad se ha categorizado en dos grupos: $>13$ años y $<13$ años. Los resultados obtenidos respecto a las diferencias según sexo y edad en actitudes e intereses en temas morales se presentan en las tablas 2 y 3 respectivamente.

Los resultados demuestran que existen diferencias estadísticamente significativas en actitudes e intereses en temas morales en función del sexo y de la edad.

En cuanto a las variables sexo y edad, en términos generales, los resultados de las diferencias de medias muestran en las chicos, en comparación con los chicas, así como en el grupo de adolescentes de menor edad, una mayor tendencia a la despenalización de la violencia si es en defensa de lo propio y menor predisposición a la emisión de conductas prosociales.

En la tabla 4 se muestran los datos correspondientes a la participación (porcentajes) de los estudiantes de Educación Secundaria en ONGs (Organizaciones No Gubernamentales) así como la realización de actividades solidarias según sexo y edad.

\section{Conclusiones y discusión}

A partir de los resultados obtenidos se puede vislumbrar, a priori, algunas tendencias tratando de conectar ítems parecidos o muy similares en lo que a su contenido se refiere, las cuales se presentan de forma resumida a continuación:

Con respecto a lo ítems 1 y 2 parece haber una respuesta bastante unánime al respecto del término robar, notándose una tendencia bastante notable hacia la categorización de robo en las situaciones propuestas, con- cretamente esto se refleja en valores del $93 \%$ y $83 \%$ respectivamente.

En los ítems 3 y 4 también parece haber una fuerte unanimidad respecto a la clasificación de un acto como violento, con porcentajes del $86 \%$ y $87 \%$ de respuestas afirmativas respectivamente. Ello puede interpretarse como cierta claridad en el alumnado de Educación Secundaria para entender una opción como violenta o como no violenta independientemente de las posibles variables y circunstancias que precipitan y envuelvan la situación. Sin embargo, se refleja cierta disparidad en cuanto a la clasificación del acto, violento o no violento, como justo o no justo, pudiéndose señalar que existe un porcentaje considerable de alumnado de Educación Secundaria que considera que un acto violento puede ser justo si es defensa de lo propio, es decir, tienen más facilidad para entender y clasificar un acto como violento que en función de la justicia que conlleva el acto en sí mismo. Ello parece ser una costumbre arraigada en nuestra cultura que supone la despenalización de la violencia si es en defensa de lo propio, con independencia de que se trate de acciones violentas o injustas en sí mismas.

En cuanto al ítem 5, a pesar de haber diferencias moderadas entre las seis opciones excluyentes del mismo, parece que el $41 \%$ atribuible a la opción número 6 permite señalar el escaso deseo del alumnado que compone la presente muestra de, a efectos prácticos, proceder ellos mismos a donar dinero a personas desconocidas porque entienden que este dinero puede ser malgastado en alcohol, drogas, etc y, por lo tanto, no sienten la necesidad de dar o contribuir con ese dinero.

En el caso del ítem 6, hay una opción ampliamente elegida $(68 \%)$ que hace referencia a que se debe sentir la necesidad de ayudar a ONGs donando dinero para colectivos desfavorecidos argumentando razones como que hay que ser solidarios y compartir los recursos. Ello refleja ya cierto grado de concienciación de este alumnado respecto a la importancia de la acción solidaria y trabajo a favor de otros.

En el ítem número 7 acerca de la cuestión de la evitación de conflictos entre allegados las respuestas se 
pueden separar entre opciones negativas y afirmativas. Concretamente, el $80 \%$ se muestran a favor del deseo de resolución de conflictos entre sus allegados y la intención de intervenir para dirimir éstos en la medida en la que les sea posible. No obstante, el restante $20 \%$ de una manera u otra manifiesta no tener la intención de evitarlo, bien porque deben arreglarse entre ellos o bien porque consideran que es mejor no estar en medio y no intervenir.

Con respecto al ítem 8 en el que se les plantea a los participantes en este estudio cuestiones referentes a si la pena de muerte puede llegar a ser justa, la mayoría, el $79 \%$ de los encuestados, se han decantado por la opción que considera la pena de muerte como no justa. Se refleja un importante acuerdo y tendencia afirmativa respecto a que la pena de muerte es injusta por parte del alumnado de Educación Secundaria.

En cuanto al ítem número 9 que trata de la dotación de viviendas por parte del Estado a personas que han perdido su hogar como consecuencia de una tragedia, $\mathrm{y}$ a tenor del porcentaje de respuestas observado, parece existir una tendencia bastante pronunciada hacia la opinión de que es una medida acertada este tipo de ayudas estatales, dándose un porcentaje del $87 \%$ a favor de las alternativas afirmativas. Parece ello reflejar una importante concienciación social por parte del alumnado de estas edades respecto a la importancia de la acción solidaria con estas personas desfavorecidas por circunstancias ajenas a su voluntad.

Con respecto al ítem referido la cuestión relativa al cierre de las fronteras a los inmigrantes parece los sujetos coincidir de forma importante en la opinión de que todos los seres humanos tienen derecho a vivir de forma digna y, por lo tanto, no se debe privar a estas personas inmigrantes de las mismas oportunidades de las que gozamos los nativos de los países más desarrollados económica y laboralmente. En cualquier caso, también es importante resaltar que un $15 \%$ de los encuestados, acerca de esta cuestión, consideran que no es conveniente la llegada masiva y descontrolada de inmigrantes para evitar posibles conflictos. En esta pregunta y sus correspondientes porcentajes de respuestas puede reflejarse el debate y controversia a nivel nacional en el que aún en la actualidad nos encontramos inmersos.

Respecto al último ítem, el ítem 11, en el que se pide que se ubiquen en la categoría de colaborador o no colaborador con una ONG, en el $23 \%$ de los casos las respuestas son afirmativas. En este sentido, la justificación de estos resultados puede encontrarse en el apartado B del ítem, en el que se proponen doce justificaciones no excluyentes sobre las razones para participar o no en una ONG. La opción más elegida con un $22.7 \%$ es la que no consideran ninguna razón concreta o no se desean precisar. La siguiente opción más elegida con un $16.1 \%$ es la respuesta afirmativa en la que se precisa que sí se colabora para ayudar a los necesitados. Las siguientes opciones más elegidas son: No, porque no conozco ninguna y No, porque no tengo tiempo, con un 12.9 y un 12.2 respectivamente. Puede destacarse que el porcentaje de estudiantes que asumen la responsabilidad y tienen creados sentimientos sobre la necesidad de realizar acciones y trabajo a favor de otros no es muy elevado, en especial, en lo que se refiere a la colaboración y ayuda con ONGs por parte del alumnado.

En conjunto, puede destacarse que el alumnado que ha participado en este estudio exhibe unos valores y actitudes en temas morales que aunque puede ser en bastantes casos apropiados y congruentes con nuestra cultura, con frecuencia se quedan en concepciones teóricas y no siempre son tenidos en cuenta ni aplicados a la práctica cotidiana, en especial, en lo que al desarrollo de acciones solidaras y trabajo a favor de otros se refiere.

El presente trabajo aporta los resultados del Cuestionario sobre Valores y Actitudes en Temas Morales, los cuáles son sumamente útiles con vistas a la educación en valores como la solidaridad. Los datos aportados por el presente estudio permiten extraer conclusiones relevantes en el actual contexto en el que cada día es mayor el número de agresiones; existiendo cada vez mayor preocupación por los problemas de convivencia social, escolar y el deterioro de las relaciones interpersonales en los centros educativos de Educación Secundaria. Un elemento importante para evaluar en estas situaciones son las actitudes e intereses en temas morales y predisposición hacia la violencia en estudiantes de educación secundaria. Por ello, en el presente estudio descriptivo se presentan los resultados de un cuestionario sobre valores y actitudes en temas morales analizando diferencias en función del sexo y de la edad; encontrándose que diferencias estadísticamente significativas por sexo y edad.

Respecto al sexo, se puede apreciar, en términos generales, una menor tendencia en las chicas a la despenalización de la violencia si es en defensa de lo propio (por ejemplo, en el ítem referido a si una persona descubre a un asaltante que había entrado a su casa a robar y le pega un tiro, las chicas consideran en mayor medida que los chicos de que se trata de una acción violenta aunque sea en defensa de lo propio; en el ítem 4 referido a si un estudiante al salir del instituto mata a otro en respuesta a una paliza que ha recibido las chicas también conceptualizan en mayor medida que los chicos dicha acción como violenta y valoran en menor medida que los chicos dicha respuesta como una acción justa). Sin embargo, las chicas muestran mayor predisposición a la emisión de conductas prosociales, sienten más necesidad de participar y colaborar con ONGs (de hecho, participan más en ONGs y afirman realizar más actividades solidarias en comparación con los chicos) y se muestran más dispuestas a intervenir si ven alguna acción injusta para alguien. Las chicas también muestran menos grado de acuerdo que los chicos ante la cuestión planteada de que Europa cierre sus 
fronteras a los inmigrantes de países con menos recursos. Estos resultados son coherentes con los obtenidos en otros estudios en los que también se encuentra mayor empatía, conductas solidarias y razonamiento moral en las chicas (Carlo y Randall, 2002; Mestre, Samper y Frías, 2002; Ortiz, Aguirrezabala, Apodaca, Etxebarría y López, 2002). En esta línea, otros estudios (Gilligan, 1985; Nunner-Winkler y Sodian, 1998) también encontraron en las chicas mayor grado de sensibilidad prosocial en estos temas así como una orientación moral más orientada a los principios de cuidado y preocupación por los otros. Puede pensarse que estos resultados pueden ser explicados por el adelanto en el desarrollo evolutivo de las chicas en comparación con los chicos o siguiendo las aportaciones consolidadas de Turiel (1994) por el impacto directo que ejercen las normas sociales y culturales en las creencias morales que inciden en el comportamiento o considerando las aportaciones de Hoffman (1987) un elemento relevante sería la influencia de los patrones morales internos promovidos en niños y niñas por sus padres y que modelan las prácticas morales más aceptable según el género. En este sentido, las pautas educativas y orientación recibida por chicos y chicas es fundamental, en consonancia con los planteamientos realizados por Zahn-Waxler, Cole y Barrett (1991) que señala que a los chicos se les ha educado orientados hacia conductas menos prosociales, por ejemplo, como instrumento para ganarse la vida en ambientes económicamente competitivos. No obstante, para finalizar este apartado puede comentarse que existen resultados contradictorios ya que mientras unos autores señalan que las chicas maduran antes que los chicos, otros encuentran que no siempre aparecen diferencias a favor de las chicas (Rest, 1979; Carlo, Roesch y Koller, 1999).

Con respecto a la edad, puede destacarse que, al igual que en el caso de las chicas, el grupo de adolescentes de mayor edad conceptualiza en mayor medida como violentas determinadas acciones como las planteadas en los ítems 3 y 4 aunque se traten de acciones en defensa de lo propio. Sin embargo, sea por un menor efecto del factor deseabilidad social u por otra razón, es el grupo de menor edad el que considera determinadas acciones como justas, por ejemplo, muestran mayor grado de acuerdo sobre el hecho de que la pena de muerte puede llegar a ser justa o sobre el hecho de que Europa cierre sus fronteras a los inmigrantes de países con menos recursos. Igual que ocurre con las chicas, es el grupo de mayor edad el que manifiesta colaborar más con ONGs o participar en mayor medida en actos de corte solidario que el grupo de menor edad. Otras investigaciones (Whiting y Edwards, 1988) también encuentran resultados similares argumentando que en la adolescencia, con independencia de la imagen que se pueda tener socialmente de este período evolutivo, disminuyen modos primitivos de razonamiento prosocial, como el razonamiento hedonista; aumentando las conductas prosociales tanto cuantitativa como cualitativamente. Estos últimos investigadores encuentran que durante la adolescencia se incrementan las capacidades de empatía, juicio moral y habilidades cognitivas que repercuten en el comportamiento prosocial y en el reconocimiento del valor de ayudar a otros siendo, por tanto, el momento ideal para la intervención psicoeducativa que mejore la convivencia en la etapa de educación secundaria. Estos datos también son congruentes con los resultados encontrados por otros autores más recientemente (De la Caba y Etxeberría, 1999) que, profundizando en la consistencia entre cognición y acción moral sobre el tema de la solidaridad en adolescentes, encuentran que la conducta moral no está tanto sujeta a la altura del juicio moral por influencias situacionales (grupo de amigos, presión del profesor, etc) sino por la propia realidad evolutiva de los adolescentes, marcada por su capacidad cognitiva formal, su búsqueda de identidad y la mayor amplitud conforme mayor sea la edad de relaciones y roles sociales desempeñados. En esta línea, las aportaciones realizadas por Aierbe, Cortés y Medrano (2001) permiten señalar que es lógico que a los 12 años la capacidad de razonamiento moral esté evolutivamente en estadios inferiores en comparación con alumnos de mayor rango de edad.

Con referencia a los temas morales, resulta llamativo que hay un grupo de alumnado que entienden que una acción violenta puede ser justa, puesto que tanto defenderse de un robo como recibir un ataque entre adolescentes activan una respuesta muy arraigada en nuestra cultura que despenaliza la violencia si es en defensa de lo propio. Este sería un tema para debate pues no cabe duda que dicha despenalización de la violencia en términos de defensa propia pertenece al ámbito personal de la moralidad (Turiel, 1983), mientras que la consideración de una acción violenta como intrínsecamente injusta pertenece al ámbito de la moral universal cuyo principal referente es la Declaración Universal de los Derechos Humanos (Schaffer, 2000). Ambos ámbitos, también denominados micromoralidad y macromoralidad respectivamente (Rest, Narváez, Bebeau y Thoma 1999a, 1999b) pueden entrar en contradicción, sobre todo en personas jóvenes que aún no han alcanzado una identidad moral que caracteriza a los adultos con nivel cultural (Blasi, 1993).

Existe una relación entre moralidad y conducta solidaria según puso de manifiesto Gilligan (Gilligan y Wiggins, 1988) al elevar a categoría moral las responsabilidades interpersonales característica del sexo femenino. Rest, Narváez, Bebeau y Thoma (1999b) entienden que la relación estriba en que mientras que la moralidad se ocupa de la macromoralidad, el comportamiento prosocial pertenece a la micromoralidad.

Esta educación en solidaridad y para la prevención de la violencia es necesaria en los centros de Educación Secundaria y en nuestras universidades ya que permite incorporar progresivamente este valor en los centros de educación secundaria pudiéndose 
emplear incluso TIC en esa transmisión y educación de valores (Morales, Infante y Galindo, 2003). Un objetivo prioritario en cuestiones de solidaridad es atacar la insolidaridad desde la educación, como instrumento de transformación social para evitar situaciones de desventaja social del tipo que sean (Consejo de Europa, 2000).

La ausencia de valores en actitudes y temas morales se relaciona con la violencia escolar en forma de agresiones las cuáles constituyen una realidad diaria en los institutos de Educación Secundaria. De hecho, numerosos estudios encuentran que una de las principales manifestaciones de esta violencia tiene lugar entre los propios adolescentes de esto centros que son los protagonistas de la mayoría de los actos violentos (Dodge, Coie, Pettit y Price, 1990; Ortega, 1995; Peiró, 2009; Prieto, 2005; Velásquez, 2005); de ahí la necesidad de una educación en valores que contribuya a la mejora de la convivencia educativa. Parece relevante señalar la importancia de evaluar estas actitudes y comportamientos que pueden entroncar y ser indicadores de un modo de proyectarse en la vida por parte de estos adolescentes que se debiera deslizar hacia un modo meramente individualista donde la sociedad es vista simplemente como un mosaico plural sin engarce entre sus miembros más allá de la competitividad pura y dura donde se confunde autonomía con individualidad. De una sociedad vertebrada en la que hay proyectos pluralistas y un modo autónomo de proyectarse en la vida, pese a su complejidad, se produce con demasiada facilidad un deslizamiento de la tolerancia a la indiferencia, es decir, la manifestación de un desentenderse de los compromisos y de la problemática del otro, de un no querer asumir la propia responsabilidad a condición de no ser interpelado o sencillamente no ser molestado en su quehacer cotidiano aún cuando desde la filosofía aristotélica se señala que vivimos en una sociedad interdependiente en la que todos nos podemos necesitar en un momento u otro. Es el riesgo de una pretendida tolerancia que no sabe discernir sus límites, respetar el compromiso ético y las exigencias de la intolerancia, deslizada hacia la indiferencia curiosamente teñida de humanitarismo.

La convivencia en la escuela se deteriora, y con ello el clima adecuado y los tan necesarios valores que intervienen en ella. Distintos organismos internacionales (ONU, UNESCO, OCDE, OEA) señalan que la educación tiene que volver su mirada a la formación de actitudes y valores en los estudiantes. Algunos autores como Ochoa, Peiró y Merma (2010) reflejan que un $16 \%$ de los docentes vienen empleando la mayor parte de su tiempo en "cuidar a los estudiantes" más que en enseñar y que además en muchas ocasiones no saben cómo afrontar las negativas actitudes de civilidad que hay en su alumnado y los conflictos que surgen. Además en alguna ocasión parece que lo importante es que el alumnado manifieste ciertas conductas aunque no estén interiorizadas ni se haya profundizado previamente en cuestiones que conllevan el despliegue de valores humanos y valores para la convivencia abordando temas como la diversidad, la igualdad, discriminación, derechos humanos y solidaridad, etc.

El comportamiento prosocial es visto hoy como una dimensión en la que se alinean, en un extremo, conductas egoístas, mientras que en el otro extremo están las conductas altruistas (Krebs y Van Hesteren, 1994). Puesto que todo acto altruista puede suponer algún beneficio para la persona puede pensarse en proponer prácticas en educación secundaria que supongan un comportamiento a favor de otros, aunque tengan utilidad para el alumnado de que la realizan.

Para concluir, se destaca la importancia de una detección y evaluación temprana de actitudes e intereses hacia estos temas morales con vistas a una educación integral dirigida a la mejora de la convivencia social y a la prevención de la violencia escolar.

\section{Extended Summary}

Values such as solidarity and non-violence should be the guiding principles of all education, influencing the development of the curriculum at the various levels of education and in pursuit of the students' comprehensive preparation. Positive attitudes and ethical values in accordance with socially desirable ethical values should be promoted, both in planning and day-to-day implementation (Trianes, 2002). The overall objective of this study is an evaluation of attitudes and interests towards moral issues in a sample of teenage middle school students.

The participants in this study were 450 middle school students, aged between 12 and 15 years old, with an average age of $13.21(S D=1.19)$, all of them from public institutions in urban areas of the province of Malaga. They were 207 boys and 243 girls, and from the results differences were analyzed based on their gender and age.

This study used the "questionnaire on values and attitudes" in moral issues (VATM). This questionnaire consists of a total of 13 items grouped in 11 areas in which the subject is given a statement or description of a conflict situation, and has to choose between various alternative responses. The questionnaire shows appropriate psychometric properties. Besides this, another version of the same questionnaire was also used, with a Likert-type response format where students evaluated the issues raised on a scale of 1 to 4 (1= strongly disagree and $5=$ strongly agree). It assesses attitudes towards issues such as personal theft, solidarity, coop- 
eration, international aid, human rights, etc. It is an attitude questionnaire to investigate these controversial issues.

With regard to the procedure, the data were collected by two counselors and a researcher from Malaga University in the various centers of secondary education which took part in this study. The questionnaire was completed in the ordinary classes, only after obtaining the relevant permissions. Students were also provided with instructions for the completion of the questionnaire, and assured of the confidentiality of the data obtained in this study, as well as that the test was purely for research purposes. The space and time set aside for the completion of the questionnaire by the students were not restricted, and students were given the opportunity to clarify any possible doubts and problems of understanding in terms of the subject or filling in of the form for the questionnaire. The replies were checked and rigorously accounted for prior to the introduction of the data in SPSS for further analysis. The statistical package SPSS was used for the statistical processing of data. Descriptive analyses reflecting percentages, averages and standard deviations were used, in addition to Student's T for independent samples, to see if there are statistically significant differences in the evaluation of attitudes and interests in moral issues based on gender and age.

The results show that a large percentage of middle school students from the sample very much agree with the aspects reflected in items 1, 4 and 7, referring to whether the situations described may be considered stealing, violent acts, or if they would try to avoid causing a conflict $(93.00 \% ; 87.00 \%$ and $80.00 \%$ respectively). $77.00 \%$ of teenage middle school students do not collaborate or participate in any $\mathrm{NGO}$; and give as their main reasons for this a lack of time or that they had not even considered it. In addtion, $71.24 \%$ are not involved in any type of solidarity activity, stating as reasons: lack of information, lack of time, laziness, the opportunity not having presented itself and never having considered it, and not having the money to do so. Many of these teenagers give as a reason the fact that they haven't considered this possibility seriously, that they have thought about it but not given importance to it, that they are unaware of any NGOs or do not have time or money to do so. The results show that there are statistically significant differences in attitudes and interests in moral issues on the basis of gender and age.

With regard to the gender and age factors, in general terms, the results of mean differences show boys and younger adolescents have a greater tendency to decriminalize violence if it is in self-defense.

With regard to gender, there is less of a tendency, in general, in girls to decriminalize violence if it is in selfdefense (for example, in the item that describes a situation where a person discovers and fires on an assailant who has entered his home to steal, the girls considered this to be a violen taction to a greater extent than boys, even if it was in self-defense; in item 4, where a situation is described in which a student kills another in response to a beating previously received, the girls also conceptualize this as a violent action to a greater extent than boys, while boys consider to a greater extent that response to be fair). The girls show a greater predisposition to prosocial behaviors. However, girls feel a greater need to participate and collaborate with NGOs (in fact, they are shown to be more involved in NGOs and claim to carry out more solidarity activities). They are also more willing to intervene on someone's behalf if they see any unjust action. The girls also show a lesser degree of agreement than the boys with regard to the issue of Europe closing its borders to immigrants from countries with fewer resources. These results are consistent with those obtained in other studies that also found greater empathy, solidarity behaviors and moral reasoning in girls (Carlo and Randall, 2002). In this sense, other studies (Gilligan, 1985;) (Nunner-Winkler and Sodian, 1998) also found greater prosocial sensitivity on these matters in girls, as well as a moral guidance geared more to the principles of care and concern for others. It may be supposed that these results can be explained by girls' relative advancement in development compared with boys, or, according to the contributions consolidated by Turiel (1994), because of the direct impact of social and cultural norms in moral beliefs that affect behavior. They could also be explained by considering contributions from Hoffman (1987), according to which the influence of the internal moral standards promoted in children by their parents would be relevant, as they shape moral practices according to gender. In this sense, educational guidelines and guidance for girls and boys are essential, in line with the approaches taken by Zahn-Waxler, Cole and Barrett (1991) that indicates that boys are educated in a way which makes them less oriented to prosocial behaviours, for example in order for them to be able to make a living in economically competitive environments.

With regard to age, it should be highlighted that the group of older teenagers have a greater tendency, just as the girls do, to conceptualize the situations described, such as those raised in items 3 and 4, as violent actions, even if they are carried out in selfdefense. However, be it due to a lesser effect of the social desirability factor or for another reason, it is the lower age group which considers certain actions as fair. For example, they show a greater degree of agreement about the fairness of the death penalty or the idea of Europe closing its borders to immigrants from countries with fewer resources. That is the case with the girls, as in the older adolescent group, who show more collaboration with NGOs and participate to a greater extent in acts of solidarity than the younger age group. Other research (Whiting and Edwards, 1988) found similar results, arguing that in 
adulthood, regardless of the image that this evolutionary period may have socially, there is a decrease in primitive modes of prosocial reasoning as hedonistic reasoning; increasing prosocial behavior both quantitatively and qualitatively. These researchers found that during adolescence the capacity for empathy, moral judgment and cognitive skills that impact on prosocial behavior and recognition of the value of helping others all increase. Thus, the ideal time for psychoeducational intervention to improve the ability to coexist is during secondary education. These data are also consistent with the results found by other authors more recently (Carlo and Randall, 2002), who investigated in more detail the consistency between cognition and moral relating to solidarity in teenagers. They fount that moral behavior is not subject to the height of moral judgment by situational influences (pressure from friends, the teacher, etc.), but rather by the developmental situation of teenagers themselves, marked by their formal cognitive ability, their search for identity and greater breadth at higher age relations and social roles held.

In conclusion, the highlighting of the importance of detection and early evaluation of attitudes and interests towards these moral issues, with a view to a comprehensive education, can contribute to the improvement of social coexistence and prevention of violence in schools.

\section{Referencias}

Aierbe, A., Cortés, A. y Medrano, C. (2001). Una visión integradora de la teoría kohlberiana a partir de las críticas contextuales: implicaciones para la educación y la investigación en el ámbito moral. Cultura y Educación, 13, 147-177.

Blasi, A. (1993). The development of identity. Some implications of moral functioning. En Noam, G. G. \& Wren, T. E. (Eds.). The moral self. Cambridge: The MIT Press.

Bolívar, A. (1995). La educación en valores y actitudes. Madrid: Grupo Anaya.

Buxarrais, M. R. (1997). La formación del profesorado en educación en valores. Propuestas y materiales. Bilbao: Desclée de Brouwer.

Carlo, G. y Randall B. A. (2002). The development of a measure of prosocial behaviors for late adolescents. Journal of Youth and Adolescence, 31 , 31-45.

Carlo, G., Roesch, S. C. y Koller, S. H. (1999). Similarities and differences in prosocial moral reasoning between Brazilian and AngloAmerican college students. Interamerican Journal of Psychology, 33, 151-172.

Consejo de Europa (2000). Sites of Citizenship Brochure. Estrasburgo: Consejo de Europa.

Cortés, L., Hernán, M. J. y López, O. (1998). Las organizaciones de voluntariado en España. Madrid: Plataforma para la Promoción del Voluntariado en España.

De la Caba, M. A. y Etchebarría, I. (1999). Consistencia entre cognición y acción Moral: un estudio con adolescentes vascos. Psicología moral y crecimiento personal. Barcelona: Ariel Psicología.

Dodge, K., Coie, J., Pettit, G. y Price, J. (1990). Peer status and aggression in boys groups: Development and contextual analyses. Child Development, 61, 1289-1309.

Fouce, J. G. (2001). Voluntarios, exvoluntarios y futuros voluntarios: un estudio descriptivo de sus perfiles y hábitos participativos. Apuntes de Psicología, 19, 519-542.

García, J. (1994). Solidaridad y voluntariado. Santander: Sal Terrae.

Gervilla, A. (1997). Estrategias didácticas para educar en valores. Madrid: Dykinson.

Gilligan, C. (1985). Psicología moral femenina. Madrid: Debate.

Gilligan, C. y Wiggins, G. (1988). The origins of morality in early childhood relationships. En C.Gilligan, J. V. Ward, \& J. M. Taylor (eds.), Mapping the Moral Domain (pp. 111-138). Cambridge, MA: Harvard University Press.

González, F. (2002). Cuaderno a bordo. Madrid: Luis Vives.

Hoffman, M. L. (1987). The contribution of empathy to justice and moral reasoning. En N. Eisenberg y J. Strayer (Eds.), Empathy and its development, (pp. 123-141). Cambridge, England: Cambridge University Press.

Krebs, D. y Van Hesteren, F. (1994). The development of altruism: toward an integrative model. Developmental Review, 14, 103-158.

Lucini, F. (1996). Temas transversales y educación en valores. Madrid: Anaya.

Mestre, V., Samper P. y Frías, M. D. (2002). Procesos cognitivos predictores de la conducta prosocial y agresiva: la empatía como factor modulador. Psicothema, 14, 227232.

Morales, F. M., Infante, L. y Galindo, A. (2003). Actitudes e intereses hacia Internet en una muestra de estudiantes de Secundaria. Encuentros en Psicología Social, 1, 3-6.

Nunner-Winkler, G. y Sodian, B. (1998). Children's un-derstanding of moral emocions, Child development, 59, 1.323-1.328.

Ochoa, A., Peiró, S. y Merma, G. (2010). Estudio comparativo de las actuaciones de los profesores de secundaria ante situaciones que alteran la convivencia escolar: el caso de Querétano (México) y Alicante (España). En J. Gázquez, y C. Pérez, (Eds.). La convivencia escolar. Aspectos psicológicos y educativos (pp.131-137). Granada: Universidad de Almería. Departamento de Psicología. Facultad de Educación: Editorial, GEU.

Ortega, R. (1995). Las malas relaciones interpersonales en la escuela: estudio sobre la violencia y el maltrato entre compañeros de segunda etapa de EGB. Infancia $y$ Sociedad, 27-28, 191-216.

Ortiz, M. J., Aguirrezabala, E., Apodaca, P., Etxbarría, I y López, E. (2002). Características emocionales, funcionamiento social y satisfacción social en escolares. Infancia $y$ aprendizaje, 25, 195-218.

Peiró, S. (2009). Valores educativos y convivencia. España: Club Universitario.

Prieto, G. (2005). Violencia escolar y vida cotidiana en una 
escuela de secundaria. Revista Mexicana de Investigación Educativa, 10, 1005-1026.

Rest, J. R. (1979). Development in judging moral issues. Minneapolis: University of Minnesota Press.

Rest, J., Narváez, D., Bebeau, M. y Thoma, S. (1999a). A neo-Kohlbergian approach to moral judgment: An overview of Defining Issues Test research. Educational Psychology Review, 11, 291-324.

Rest, J. R., Narváez, D., Bebeau, M. y Thoma, S. (1999b). Postconventional moral thinking: A neo-Kohlbergian approach. Mahwah, NJ: Erlbaum.

Schaffer, H. R. (2000). Desarrollo social. México: Siglo XXI Editores.

Touriñán, J. M. (2008). Educación en valores, educación intercultural y formación para la convivencia pacífica. La Coruña: Netbiblo.

Trianes, M. V. y Fernández, C. (2001). Aprender a ser personas y a convivir. Un programa para secundaria. Bilbao: Desclée de Brouwer.

Trianes, M. V. (2002). Cuestionario sobre Valores y Actitudes en Temas Morales. Extraído el 10 Julio, 2002, de los Apuntes de Psicología de la Educación y sus prácticas de la Plataforma virtual de la UMA.

Turiel, E. (1983). The Development of Social Knowledge: Morality and Convention. Cambridge: Cambridge University Press.

Turiel, E. (1994). Morality, authoritarianism, and personal agency in cultural contexts. En R. J. Sternberg y P. Ruzgis (Eds.), Personality and intelligence (pp. 271-302). Cambridge, England: Cambridge University Press.

Velásquez, L. (2005). Experiencias estudiantiles con la violencia en la escuela. Revista Mexicana de Investigación Educativa, 10, 739-764.

Whiting, B. B. y Edwards, C. P. (1988). Children of different worlds: The formation of social behavior. Cambridge, MA: Harvard University Press.

Zahn-Waxler, C., Cole, P. M. y Barrett, K. C. (1991). Guilt and empathy: Sex differences and implications for the development of depression. En J. Garber y K. A. Doge (Eds.), The development of emotion regulation and dysregulation (pp. 243-272). New York: Cambridge University Press.

Manuscrito recibido: 24/08/2011

Revisión recibida: 07/11/2011

Manuscrito aceptado: 12/11/2011 